\title{
Modeling and Analysis of Multi-path Effects in Static and Dynamic Sea Surface
}

\author{
Yuanming DING ${ }^{1,2, a}$, Shiguang WANG ${ }^{2,3}$ and Chengsheng PAN ${ }^{1,2}$ \\ ${ }^{1}$ Network Technology Engineering Laboratory of Smart Shipping and Logistics \\ ${ }^{2}$ Key Laboratory of Communication Networks and Information Processing \\ ${ }^{3}$ College of Information Engineering, Dalian UniversityNo.10, Xuefu Street \\ JinZhou Xinqu, Dalian 116622, P. R. China \\ aemail: dingyuanming@dlu.edu.cn
}

Keywords: Multi-path effect, Modeling, Distance difference, Rough sea surface

\begin{abstract}
For the current multi-path effects geometric modeling lack of considering the impact of the earth curvature, sea surface multi-path model under the Earth curvature is analyzed. By geometry models, the static sea surface multi-path effects and dynamic sea surface environment are described, and the equation of distance difference caused by multi-path effects is deduced. Based on the equation, the simulation under the receiving antenna reflection point in the range of $1-50 \mathrm{~m}$ is carried out. The simulation results show that, for static sea, when the distance between the reflection point and receiving antenna is less than about $5 \mathrm{~m}$, the greater satellite elevation the greater distance difference; when the distance between the reflection point and receiving antenna is more than about $7 \mathrm{~m}$, the smaller satellite elevation the greater distance difference. For dynamic sea surface, the smaller satellite elevation and the greater waves grade, the greater distance difference caused by multi-path effects.
\end{abstract}

\section{Introduction}

Satellite's downlink signal will be refracted and reflected by the sea when the high orbit satellite and the maritime mobile equipment communicate. The signal is divided into the direct signal and reflected signal. The superposition signal entered the receiving antenna of the maritime mobile equipment, thus affecting the communication between the satellite and the maritime mobile devices, and affecting the establishment of communication link, and then the deviation caused by the multi-path effect is produced. According to the different reflection environment, the reflection can be divided into the surface reflection and the diffuse reflection ${ }^{[1]}$. When the height of the satellite transmission spot is lower and the reflection spot is close to the receiving spot, the surface of the earth can be regarded as a horizontal reflector. When the distance of the satellite sending end to the reflection surface and the receiving antenna is further, the effects of the earth's curvature cannot be ignored. So the establishment of multi-path model must be adopted in the diffuse reflection model ${ }^{[2]}$.

Recently, domestic and foreign scholars have carried out some related research in the multi-path modeling. According to their research the multi-path model can basically be divided into three kinds. The first kind is the surface reflecting, meeting the specular reflection formula. This is the most fundamental and widely used model. The second kind is the diffuse reflection which the reflective surface is rough, such as the ground, the ups and downs of the rough sea surface. This reflecting surface is a curved surface. The total of the direct signal and multi-path signal cannot satisfy a definite geometric relationship for the amplitude and phase of multi-path signal is random. As a result, to establish the multi-path model is complex. The third based on the consideration of the effect of earth curvature in the above two models. Literature [3] simulated the process of multi-path effect in the environment of calm and dynamic lake surface; Cohen and Parkinson et al., through establishing the function model of multi-path and satellite elevation angle to correct the multi-path effect of the satellite [4]; in [5], the sea surface multi-path model of meter band is studied. However, 
the researches on the geometric model of the sea surface multi-path effects are less considered of the earth curvature effect. Nevertheless, the curvature of the earth and the dynamic surface cannot be ignored when the transmitter is too far away. Therefore, the curvature of the earth is considered to establish a dynamic sea multi-path effects model, to provide technical support for the analysis of wave impact on satellite communications.

\section{Sea Surface Multi-Path Model Considering the Earth Curvature}

Hajj has put forward the basic principle that established the multi-path effect model in early $1990^{[8]}$. This paper follows this basic principle and the influence of earth curvature on the multi-path effect must be considered when the satellite communication distance is very far from reflector. So this paper researches from two aspects, the first is multi-path effect model of static sea surface with considering the earth curvature, the second is multi-path effect model of dynamic sea surface with considering the earth curvature.

\section{Static Sea Surface Multi-path Modeling Considering Curvature of the Earth}

It can be considered that signals reaching the antenna and the sea surface from same satellite are parallel if the satellite is very far away from the reflection area compared with the antenna [5]. The reflection is regarded as the mirror reflection, meeting the law of reflection. The multi-path effect model as be shown in Figure. 1.

The mirror reflection is occurred at the point of $z\left(\mathrm{x}_{1}, 0\right), H_{r}$ is receiving antenna height, $H_{t}$ is the transmitter height, $G$ is the ground curved distance which transmitter to the receiver. $R_{e} \approx 6379 \cdot 4 / 3 \approx 8349(\mathrm{~m})^{[9]}, R_{e}$ is the equivalent radius of the earth. $\theta$ is the satellite elevation angle when multi-path effects occur. Therefore, according to the Fig. $1, S_{d}$ can be calculated. Through $S_{d}$, the phase difference can be obtained, and then the multi-path fading signals can be analyzed.

According to the geometric model, the equivalent height of the receiving antenna and the equivalent height of the satellite transmitter can be derived ${ }^{[11]}$ :

$H_{r}^{\prime} \approx H_{r}-\frac{G_{1}^{2}}{2 R_{e}}, \quad H_{t}^{\prime} \approx H_{t}-\frac{\left(G-G_{1}\right)^{2}}{2 R_{e}}$

Where, the curved distance $G_{1}$ between the receiving antenna and the reflection point can be obtained by the following equation. $2 G_{1}^{3}-3 G G_{1}^{2}+\left[G^{2}-2 R_{e}\left(H_{r}+H_{t}\right)\right] G_{1}+2 R_{e} G H_{e}=0$

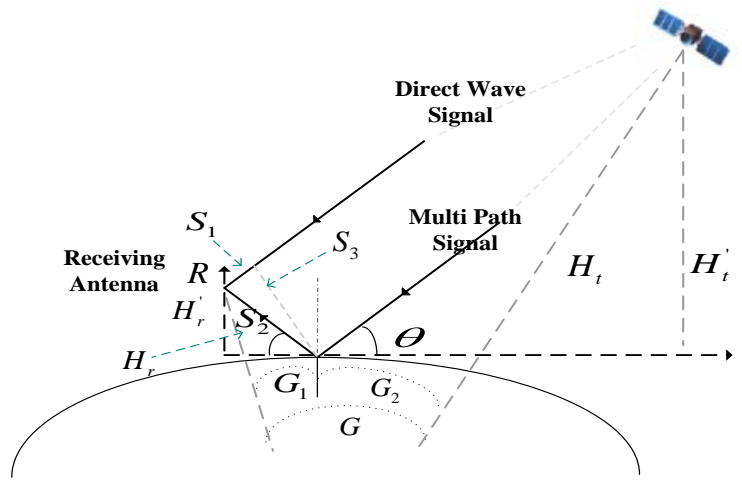

Fig.1. Static sea surface multi-path effect model considering the curvature of the Earth

In Fig. 1, can be obtained: $S_{2}=\frac{H_{r}^{\prime}}{\sin \theta}, \quad S_{3}=\frac{\left|x_{1} \tan \theta+H_{r}^{\prime}\right|}{\sqrt{\tan ^{2} \theta+1}}$

From the above formula: $S_{1}=\sqrt{S_{2}^{2}-S_{3}^{2}}$. Then, $S_{d}$ can be derived:

$$
S_{d}=S_{2}-S_{1}=\frac{H_{r}-\frac{G_{1}^{2}}{2 R_{e}}}{\sin \theta}-\sqrt{\frac{\left(H_{r}-\frac{G_{1}^{2}}{2 R_{e}}\right)^{2}}{\sin ^{2} \theta}-\frac{\left(x_{1} \tan \theta+H_{r}-\frac{G_{1}^{2}}{2 R_{e}}\right)^{2}}{\tan ^{2} \theta+1}}
$$




\section{Dynamic Sea Surface Multi-path Model Considering Curvature of the Earth}

In this paper, the plane linear sea wave model is adopted to simulate the sea wave $y=A \cos (\omega x+\varphi)$ ${ }^{[11]}$. According to the Rayleigh criterion, the sea surface will no longer be regarded as a static sea in 4 or 4 above sea-level. And the reflection is regarded as diffuse reflection or curved reflection. Figure 2 is the dynamic sea surface multi-path effect model considering the curvature of the earth. When the satellite signals arrive to the dynamic sea surface, the height of the multi-path reflection point is be supposed as $\mathrm{z}(\mathrm{x} 1, \mathrm{y} 1)$. The multi-path effect model is as shown in Fig2.

According to the static model, it can be obtained:

$$
H_{r}^{\prime} \approx\left(H_{r}-y_{1}\right)-\frac{G_{1}^{2}}{2\left(R_{e}+y_{1}\right)}, \quad H_{t}^{\prime} \approx\left(H_{t}-y_{1}\right)-\frac{\left(G-G_{1}\right)^{2}}{2\left(R_{e}+y_{1}\right)}
$$

In Fig. 2, According to the geometric relations as well as conversion of surface coordinate and plane coordinate ${ }^{[7-8]}$, it can be obtained: $\psi_{1}=\psi_{2}, \psi_{1}=\frac{\pi}{2}-(\theta-\beta), \psi_{2}=\frac{\pi}{2}-\left(\arctan \frac{H_{r}^{\prime}}{x_{1}}+\beta\right)$

Where, $\beta=\arctan \left(-A \omega \sin \left(x_{1}+\varphi_{1}\right)\right)$

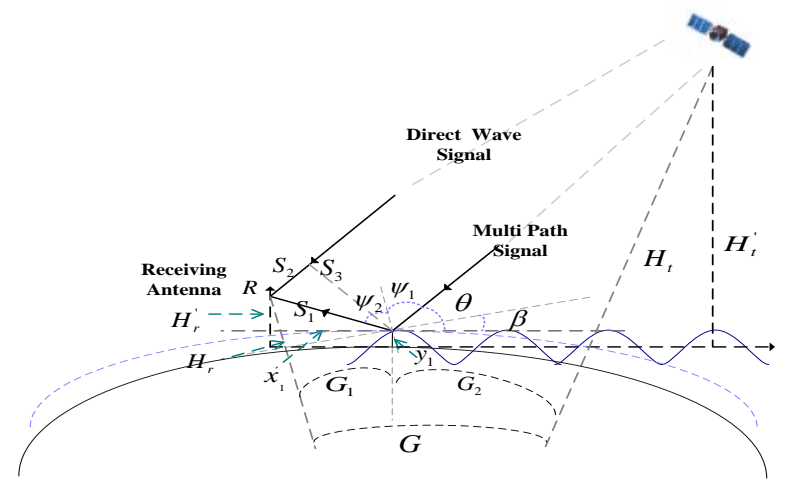

Fig.2. Dynamic sea surface multi-path effect model considering the curvature of the Earth

According to $\psi_{1}=\psi_{2}$, it can be obtained: $\theta-2\left(\arctan \left(-A \omega \sin \left(x_{1}+\varphi_{1}\right)\right)\right)=\arctan \frac{H_{r}^{\prime}}{x_{1}}$

In fig. $2, S_{1}$ the distance from the reflection point to the receiving antenna is:

$$
s_{1}=\sqrt{x_{1}^{2}+\left(\left(H_{r}-A \cos \left(\omega x_{1}+\varphi_{1}\right)\right)-\frac{G_{1}^{2}}{2\left(R_{e}+A \cos \left(\omega x_{1}+\varphi_{1}\right)\right)}\right)^{2}}
$$

According to the point to line distance formula, $S_{3}$ is:

$$
s_{3}=\frac{\left|x_{1} \tan \theta+\left(H_{r}-A \cos \left(\omega x_{1}+\varphi_{1}\right)\right)-\frac{G_{1}^{2}}{2\left(R_{e}+A \cos \left(\omega x_{1}+\varphi_{1}\right)\right)}\right|}{\sqrt{\tan ^{2} \theta+1}}
$$

According to the Pythagorean Theorem of right angled triangle, can obtain $S_{2}=\sqrt{S_{1}^{2}-S_{3}^{2}}$. The formula of the multi-path distance difference $S_{d}$ can be derived:

$$
\begin{aligned}
S_{d}=\sqrt{x_{1}^{2}+\left(\left(H_{r}-A \cos \left(\omega x_{1}+\varphi_{1}\right)\right)-\frac{G_{1}^{2}}{2\left(R_{e}+A \cos \left(\omega x_{1}+\varphi_{1}\right)\right)}\right)^{2}} & -\sqrt{x_{1}^{2}+\left(\left(H_{r}-A \cos \left(\omega \varphi_{1}+\varphi_{1}\right)\right)-\frac{G_{1}^{2}}{2\left(R_{e}+A \cos \left(\omega \varphi_{1}+\varphi_{1}\right)\right)}\right)^{2}-\frac{\left(x_{1} \tan \theta+\left(H_{r}-A \cos \left(\omega \varphi_{1}+\varphi_{1}\right)\right)-\frac{G_{1}^{2}}{2\left(R_{e}+A \cos \left(\omega \varphi_{1}+\varphi_{1}\right)\right)}\right)^{2}}{\tan ^{2} \theta+1}}
\end{aligned}
$$




\section{Simulation Analysis}

\section{Distance Difference of the Static Surface Multi-Path Effect Considering Earth Curvature}

The simulation results are shown in Fig. 3 (a) and (b). In Fig.3 (a), the satellite elevation angle is constant, $\theta=40^{\circ}$, in Fig. 3 (b), the receiving antenna height is constant and $H_{r}=2 \mathrm{~m}$.

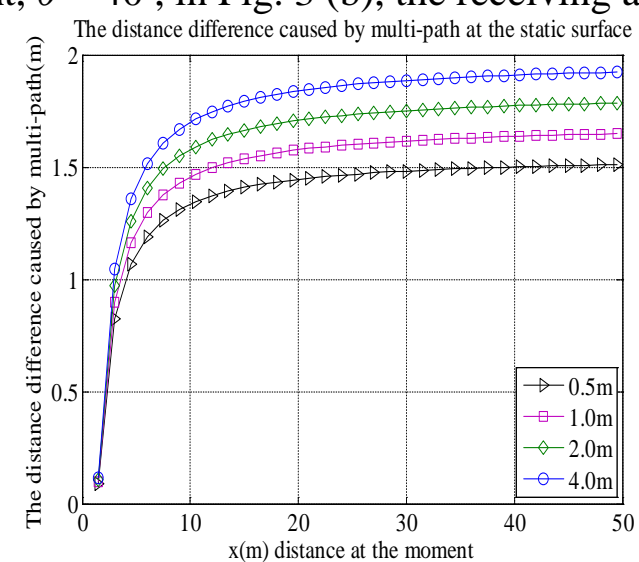

(a) The satellite elevation angle $\theta=40^{\circ}$

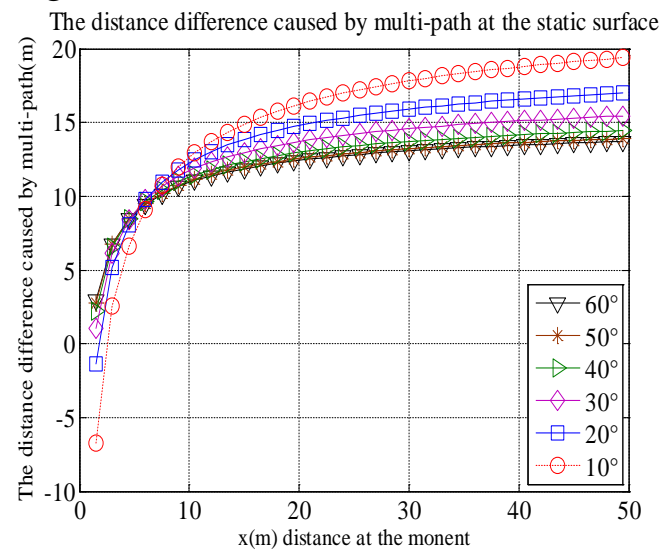

(b) The receiving antenna height $H_{r}=2 \mathrm{~m}$

Fig.3. Distance difference caused by the static sea surface multi-path effect

The simulation results in Fig. 3 show that the distance $S_{d}$ caused by the multi-path effect will be decided by the satellite elevation angle $\theta$ and the receiving antenna height $H_{r}$. In Fig. 3 (a), the distance difference caused by the multi-path effect will be increased with the receiving antenna height when the satellite elevation angle is constant, but the gap between them is very small; and this distance difference will be increased with the distance of $x$. The distance difference aroused from the receiving antenna height changes in same horizontal line when $x$ is farther, so the main factors is not the receiving antenna height. In Fig. 3 (b) the distance difference caused by the multi-path effect will be increased with increase of the satellite elevation angle when the receiving antenna height is constant, and the reflection point from the antenna is less than $5 \mathrm{~m}$. Then the distance difference caused by the multi-path effect will be increased with the increase of $x$ under each satellite angles; the distance difference caused by the multi-path effect will be decreased with increase of the satellite elevation angle when the reflection point to the receiving antenna is more than $7 \mathrm{~m}$, and the distance difference caused by the multi-path effect will also be increased with the increase of $x$ under each satellite angles. Comprehensively, the satellite elevation angle and the distance of the reflection point to the receiving antenna have a certain influence to the distance difference caused by multi-path effect.

\section{Distance Difference of the Dynamic Surface Multi-Path Effect Considering Earth Curvature}

Due to the main influence factors is not the receiving antenna height, only the effect of satellite elevation angle and the sea wave level to the distance difference caused by the multi-path effect are considered. In the simulation, the plane linear sea wave model is adopted to simulate the sea wave $y=A \cos (\omega x+\varphi)$. In Fig. 4 (a), the receiving antenna height $H_{r}=2 \mathrm{~m}$, the satellite elevation angle $\theta=40^{\circ}$. In Fig. 4 (b) the receiving antenna height $H_{r}=2 \mathrm{~m}$, the sea-level grade is 4 , the amplitude of $A=1.25$, the frequency $\omega=1.25$, the initial phase $\varphi=0$. The simulation results are shown in the Fig. 4 (a) (b). 


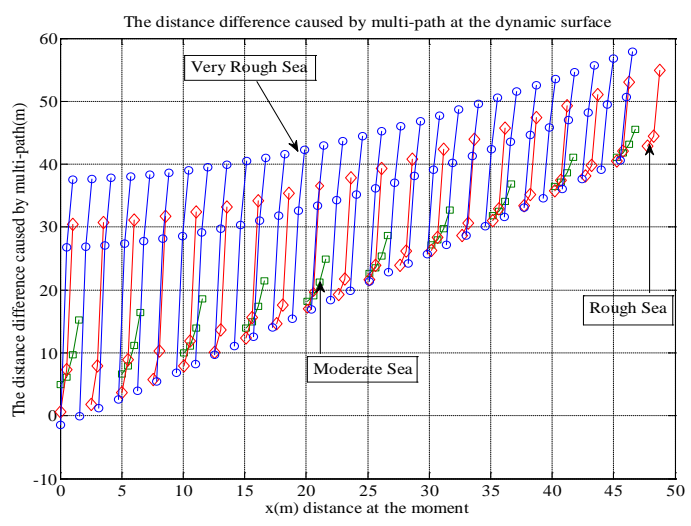

(a) Satellite elevation angle $\theta=40^{\circ}$

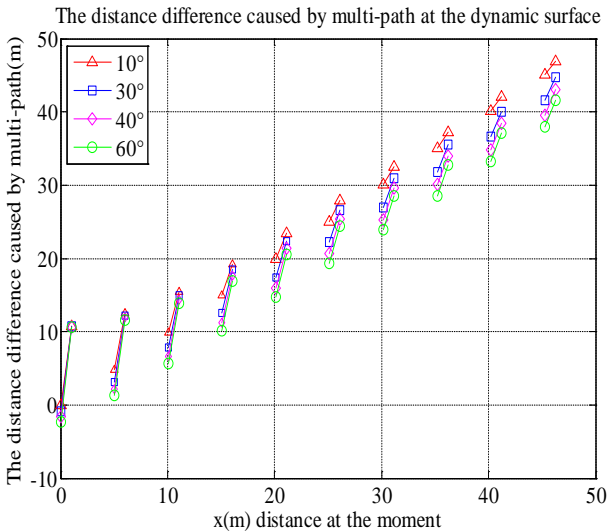

(b) Sea-level grade 4 waves

Fig.4. Distance difference caused by the dynamic sea surface multi-path effect

From the theory analysis of Fig. 2 as well as Fig. 4 (a) and (b), the reflection will not occur when the signals pass through the falling edge of the sea wave, only at the rising edge of the sea wave, the multi-path effect will be occurred. Figure 4 (a) shows that when the receiving antenna height and satellite elevation angle is constant, the distance difference caused by the multi-path effect will increase with the sea level, and the distance has an upward trend with the increase of $x$ at the same sea level. In Fig. 4 (b), the distance difference caused by the multi-path effect is almost the same in the different satellite elevation angles when the receiving antenna height is constant and the reflection point from the antenna is about $15 \mathrm{~m}$. But when the distance is more than $15 \mathrm{~m}$, different satellite elevation angles will cause different multi-path effects. The distance difference caused by the multi-path effect is bigger and has an upward trend with the increase of $x$ when the satellite elevation angle gets smaller. As the simulation analysis, the satellite elevation angle and the sea level are the main factors to the distance difference caused by the multi-path effect.

\section{Conclusions}

Through the research, the sea surface has the strongest reflection effect on satellite signal, and is the most easily to produce multi-path effect. In this paper the model is designed with considering the Earth curvature under the static sea surface and dynamic sea surface environment separately. And the equation of distance is deduced in the two conditions. The simulation results show that for static sea, in a certain antenna height, when the distance between the reflection point and receiving antenna less than about $5 \mathrm{~m}$, the distance difference caused by multi-path effects increases with the satellite elevation increases; when the distance between the reflection point and receiving antenna more than about $7 \mathrm{~m}$, the distance difference decreases with the satellite elevation increases. For dynamic sea surface, when the antenna height and the waves grade in certain condition, the distance difference caused by multi-path effects decreases with the satellite elevation increases; when the antenna height and the satellite elevation in certain condition, the greater the waves grade the greater the distance difference caused by multi-path effects. So, the smaller the satellite elevation and the greater the waves grade, the greater the distance difference caused by multi-path effects. This article has important practical value for the sea surface multi-path effects research.

\section{Acknowledgment}

This work was supported by Program for Liaoning Innovative Research Team in University (No. LT2014025) and Scientific Research Project of Liaoning Province Educational Committee (No. L 2012440).

\section{References}

[1] Ann S., Yang E., Chun J., et al. Low Angle Tracking using Iterative Multipath Cancellation in Sea Surface Environment [C]. Radar Conference, 2010 IEEE, 2010: 1156-1160. 
[2] Skolnik M.. Radar Handbook [M]. New York: McGraw-Hill. 1990, 699-810.

[3] Liu YY, Dang YM, Zhang SH. Preliminary discussion of multipath effect model on sea surface [J].Science of Surveying and Mapping. 2013; 38(1): 122-124.

[4] Cohen C., Parkinson B. Mitigating Multipath Error in GPS based Attitude Determination [J]. Guidance and Control Advances in Astronautically Sciences. 1991, 74: 53-68

[5] Dong M, Zhao YB, Zhang SH. The Analysis of the Multipath Model under the VHF Band at Sea [J]. Acta Electtronic Sinica. 2009; 36(6): 1373-1377.

[6] Peng SR. The Elimination of GPS Multipath Error reconstructs Correlation Curve Method [J]. Journal of Dalian University of Technology.2008, 3 (48): 441-445.

[7] Xie F, Zhang ZC, Zhang P, Li BZ. Influence on direction finding error by multipath effect [J]. Electronic Measurement Techology.2010, 1 (33): 29-33.

[8] Cheng Wei. Study on Some Problems of Height Fitting of GPS [J]. Surveying and Mapping of Geology and Resources. 2003, 9(4):0-12.

[9] Yang Y, Wang XS, Zhang WM, Xiao SP. Detection Technology of Low-flying Target on the Sea in Multipath Environment [J]. Journal of electronics and information. 2011 (33): 1779-1785.

[10]Yang XK , Yang XL. New Elevation Calculation Formula of Satellite Receiving Antenna[J]. Cable TV Technology. 2010; 1: 38-41.

[11]Wang YY, Liu Z, Cao YH. The Algorithm Base on the Compressed Sensing Low Altitude Radar [J]. Journal of Systems engineering and Electronic technology.2014, 4 (36): 667-671.

[12]Wang L., Sun X., Lu K. A Maximum-entropy Compound Distribution Model for Extreme Wave Heights of Typhoon-affected Sea Areas [J]. China Ocean Eng., 2012, 26(1): 49-58. 